\title{
Free Boundary Value Problem for the One-Dimensional Compressible Navier-Stokes Equations with Density-Dependent Viscosity and Discontinuous Initial Data
}

\author{
Ruxu Lian ${ }^{1,2}$ and Guojing Zhang ${ }^{3}$ \\ ${ }^{1}$ College of Mathematics and Information Science, North China University of Water Resources and Electric Power, \\ Zhengzhou 450011, China \\ ${ }^{2}$ Institute of Atmospheric Physics, Chinese Academy of Sciences, Beijing 100029, China \\ ${ }^{3}$ School of Mathematics and Statistics, Northeast Normal University, Changchun 130024, China
}

Correspondence should be addressed to Ruxu Lian; ruxu.lian.math@gmail.com

Received 9 February 2013; Accepted 6 June 2013

Academic Editor: Nazim Idrisoglu Mahmudov

Copyright (C) 2013 R. Lian and G. Zhang. This is an open access article distributed under the Creative Commons Attribution License, which permits unrestricted use, distribution, and reproduction in any medium, provided the original work is properly cited.

We study the free boundary value problem for one-dimensional isentropic compressible Navier-Stokes equations with densitydependent viscosity coefficient and discontinuous initial data in this paper. For piecewise regular initial density, we show that there exists a unique global piecewise regular solution, the interface separating the flow and vacuum state propagates along particle path and expands outwards at an algebraic time-rate, the flow density is strictly positive from blow for any finite time and decays pointwise to zero at an algebraic time-rate, and the jump discontinuity of density also decays at an algebraic time-rate as the time tends to infinity.

\section{Introduction}

In the present paper, we consider the free boundary value problem to one-dimensional isentropic compressible NavierStokes equations with density-dependent viscosity coefficient for piecewise regular initial data connected with the infinite vacuum via jump discontinuity. In general, the onedimensional isentropic compressible Navier-Stokes equations with density-dependent viscosity coefficient read

$$
\begin{gathered}
\rho_{t}+(\rho u)_{x}=0, \\
(\rho u)_{t}+\left(\rho u^{2}+P(\rho)\right)_{x}=\left(\mu(\rho) u_{x}\right)_{x}, \quad(x, t) \in R \times[0, T],
\end{gathered}
$$

where $\rho>0$ and $u$ denote the flow density and velocity, respectively, the pressure-density function is taken as $P(\rho)=$ $\rho^{\gamma}$ with $\gamma>1$, and the viscosity coefficient is $\mu(\rho)=\rho^{\alpha}$ with $\alpha>0$. Note here that the case $\gamma=2$ and $\alpha=1$ in (1) corresponds to the viscous Saint-Venant system.

There is huge literature on the studies of the global existence of weak solutions and dynamical behaviors of jump discontinuity for the compressible Navier-Stokes equations with discontinuous initial data; for example, as the viscosity coefficients are both constants, the global existence of discontinuous solutions of one-dimensional Navier-Stokes equations was derived by Hoff [1-3]. Hoff investigated the construction of global spherically symmetric weak solutions of compressible Navier-Stokes equations for isothermal flow with large and discontinuous initial data [4]; therein it is also proved that the discontinuities in the density and pressure persist for all time, convecting along particle trajectories and decaying at a rate inversely proportional to the viscosity coefficient. The global existence theorems for the multidimensional Navier-Stokes equations of isothermal compressible flows with the polytropic equation of state $p(\rho)=\rho^{\gamma}(\gamma \geq$ 1) were also showed by Hoff $[5,6]$. Chen et al. obtained the global existence of weak solutions for the Navier-Stokes equations for compressible, heat-conducting flow in one space dimension with large, discontinuous initial data [7]. Hoff showed the global existence of weak solutions of the Navier-Stokes equations for compressible, heat-conducting 
fluids in two and three space dimensions, when the initial data may be discontinuous across a hypersurface of $R^{n}$ [8]. The global existence of solutions of the Navier-Stokes equations for compressible, barotropic flow in two space dimensions which exhibit convecting singularity curves was also proved by Hoff [9].

If the viscosity coefficients $\mu(\rho)=\rho^{\alpha}, \lambda(\rho)=0$, for the case of one space dimension, the global existence of unique piecewise smooth solution to the free boundary value problem was obtained by Fang-Zhang for (1) with $0<\alpha<1$, where the initial density is piecewise smooth with possibly large jump discontinuities [10]. Lian et al. considered the initial boundary value problem for (1) with $0<\alpha \leq 1$ subject to piecewise regular initial data with initial vacuum state included in [11]. Lian et al. also addressed the Cauchy problem for one-dimensional isentropic compressible Navier-Stokes equations with density-dependent viscosity coefficient [12]; in these two cases above, they proved the global existence of unique piecewise regular solution and the finite time vanishing of vacuum state was proved in [11]. In particular, they got that the jump discontinuity of density decays exponentially but never vanishes in any finite time and the piecewise regular solution tends to the equilibrium state as $t \rightarrow+\infty$.

Recently, there are also many significant progresses achieved on the compressible Navier-Stokes equations with density-dependent viscosity coefficients. For instance, the mathematical derivations are obtained in the simulation of flow surface in shallow region $[13,14]$. The good-posedness of solutions to the free boundary value problem with initial finite mass and the flow density being connected with the infinite vacuum either continuously or via jump discontinuity was considered by many authors; refer to [15-22] and references therein. The global existence of classical solutions is shown by Mellet and Vasseur [23]. The qualitative behaviors of global solutions and dynamical asymptotics of vacuum states are also made, such as the finite time vanishing of finite vacuum or asymptotical formation of vacuum in longtime, the dynamical behaviors of vacuum boundary, the longtime convergence to rarefaction wave with vacuum, and the stability of shock profile with large shock strength; refer to [24-28] and references therein.

In this present paper, we consider the free boundary value problem (FBVP) for one-dimensional isentropic compressible Navier-Stokes equations and focus on the existence, regularities, and dynamical behaviors of global piecewise regular solution, and so forth. As $\gamma>1,0<\alpha \leq 1$, we show that the free boundary value problem with piecewise regular initial data admits a unique global piecewise regular solution, the interface separating the flow and vacuum state propagates along particle path and expands outwards at an algebraic time-rate, the flow density is strictly positive from blow for any finite time and decays pointwise to zero at an algebraic, and the jump discontinuity of density also decays at an algebraic time-rate as $t \rightarrow+\infty$ (refer to Theorem 2 for details).

The rest part of the paper is arranged as follows. In Section 2, the main results about the existence and dynamical behaviors of global piecewise regular solution for compressible Navier-Stokes equations are stated. Then, some important a priori estimates will be given in Section 3. Finally, the theorem is proved in Section 4.

\section{Main Results}

We are interested in the global existence and dynamics of the free boundary value problem for (1) with following initial data and boundary conditions:

$$
\begin{gathered}
(\rho, u)(x, 0)=\left(\rho_{0}, u_{0}\right), \quad x \in\left[0, a_{0}\right], \\
u(0, t)=0, \quad\left(\rho^{\gamma}-\rho^{\alpha} u_{x}\right)(a(t), t)=0, \quad t>0,
\end{gathered}
$$

where $x=a(t)$ is the free boundary defined by

$$
\frac{d}{d t} a(t)=u(a(t), t), \quad a(0)=a_{0}, \quad t>0 .
$$

Next, we give the definition of weak solution to the free boundary problem (1) and (2).

Definition 1 (weak solution). For any $T>0,(\rho, u)$ is said to be a weak solution of the free boundary problems (1) and (2), if $(\rho, \sqrt{\rho} u)$ has the following regularities:

$$
\begin{gathered}
0 \leq \rho \in L^{\infty}\left(0, T ; L^{1}([0, a(t)]) \cap L^{\gamma}([0, a(t)])\right), \\
\sqrt{\rho} u \in L^{\infty}\left(0, T ; L^{2}([0, a(t)])\right), \\
\left(\rho^{\gamma}-\rho^{\alpha} u_{x}\right) \in L^{2}\left(0, T ; H^{1}([0, a(t)])\right),
\end{gathered}
$$

and (1) is satisfied in the sense of distributions. Namely, it holds for all $\varphi \in C_{0}^{\infty}([0, a(t)] \times[0, T))$ that

$$
\begin{gathered}
\int_{0}^{a_{0}} \rho_{0} \varphi(x, 0) d x+\int_{0}^{T} \int_{0}^{a(t)} \rho \varphi_{t} d x d t \\
+\int_{0}^{T} \int_{0}^{a(t)} \sqrt{\rho} \sqrt{\rho} u \varphi_{x} d x d t=0
\end{gathered}
$$

and for all $\psi \in C_{0}^{\infty}([0, a(t)] \times[0, T))$ that

$$
\begin{aligned}
& \int_{0}^{a_{0}} \rho_{0} u_{0} \psi(x, 0) d x \\
& \quad+\int_{0}^{T} \int_{0}^{a(t)}\left(\sqrt{\rho} \sqrt{\rho} u \psi_{t}+(\sqrt{\rho} u)^{2} \psi_{x}\right) d x d t \\
& +\int_{0}^{T} \int_{0}^{a(t)}\left(\rho^{\gamma}-\rho^{\alpha} u_{x}\right) \psi_{x} d x d t=0 .
\end{aligned}
$$

For simplicity, we consider the initial data in FBVP (1) and (2) with one discontinuous point $y_{0} \in\left(0, a_{0}\right)$; namely, for some constant $\rho_{-}>0$

$$
\begin{gathered}
\inf _{\left[0, y_{0}\right) \cup\left(y_{0}, a_{0}\right]} \rho_{0} \geq \rho_{-}>0, \\
\left(\rho_{0}, u_{0}\right) \in W^{1, \infty}\left(\left[0, y_{0}\right) \cup\left(y_{0}, a_{0}\right]\right), \\
\left(\rho_{0}^{\gamma}-\rho_{0}^{\alpha} u_{0 x}\right)\left(a_{0}\right)=0, \\
\rho_{0}\left(y_{0}-0\right)>\rho_{0}\left(y_{0}+0\right),
\end{gathered}
$$


and the compatibility conditions between initial data and boundary conditions hold.

We will give the global existence and time-asymptotic behavior of piecewise regular solution as follows.

Theorem 2. Let $\gamma>1$ and $0<\alpha \leq 1$. Assume that the initial data satisfies (7). Then, there exists a unique global piecewise regular solution $(\rho, u, a)$ to the FBVP (1) and (2) satisfying for $T>0$

$$
\begin{aligned}
& \rho \in L^{\infty}\left(0, T ; H^{1}([0, y(t)) \cup(y(t), a(t)])\right) \\
& \cap C^{0}([0, y(t)) \cup(y(t), a(t)] \times[0, T]), \\
& u \in L^{\infty}\left(0, T ; H^{1}([0, a(t)])\right) \\
& \cap L^{2}\left(0, T ; H^{2}([0, a(t)])\right), \\
& u_{t} \in L^{2}\left(0, T ; L^{2}([0, a(t)])\right), \\
& a(t) \in H^{1}([0, T]), \\
&\left(\rho^{\gamma}-\rho^{\alpha} u_{x}\right) \in L^{\infty}\left(0, T ; L^{2}([0, y(t)) \cup(y(t), a(t)])\right),
\end{aligned}
$$

where $x=y(t)$ is a curve defined by

$$
\frac{d y(t)}{d t}=u(y(t), t), \quad y(0)=y_{0}, t>0
$$

along which the Rankine-Hugoniot conditions hold

$$
[u(y(t), t)]=0, \quad\left[\rho^{\gamma}(y(t), t)\right]=\left[\rho^{\alpha} u_{x}(y(t), t)\right],
$$

where $[f(y(t), t)]:=f(y(t)+0, t)-f(y(t)-0, t)$, and along the discontinuity $r=\xi(t)$ the jump satisfies

$$
\left|\left[\rho_{0}^{\alpha}\left(y_{0}\right)\right]\right| e^{-C_{0} t} \leq\left|\left[\rho^{\alpha}(y(t), t)\right]\right|,
$$

where $C_{0}$ is a positive constant independent of time.

If it further holds that $u_{0} \in H^{2}\left(\left[0, a_{0}\right]\right)$, then $(\rho, u, a, b)$ satisfies

$$
\begin{gathered}
\rho \in L^{\infty}\left(0, T ; H^{1}([0, y(t)) \cup(y(t), a(t)])\right), \\
\rho_{t} \in L^{\infty}\left(0, T ; L^{2}([0, y(t)) \cup(y(t), a(t)])\right), \\
u \in L^{\infty}\left(0, T ; H^{1}([0, a(t)])\right) \\
\cap L^{2}\left(0, T ; H^{2}([0, a(t)])\right), \\
u_{t} \in L^{\infty}\left(0, T ; L^{2}([0, a(t)])\right) \\
\cap L^{2}\left(0, T ; H^{1}([0, a(t)])\right), \\
a(t) \in H^{2}([0, T]), \\
\left(\rho^{\gamma}-\rho^{\alpha} u_{x}\right) \in L^{\infty}\left(0, T ; H^{1}([0, y(t)) \cup(y(t), a(t)])\right) .
\end{gathered}
$$

The domain expands outwards at an algebraic rate in time as

$$
C(1+t)^{\gamma /(\gamma-\alpha)} \geq a(t) \geq \begin{cases}c(1+t), & 1<\gamma<2 \alpha, \\ c(1+t)^{1-\gamma}, & \gamma=2 \alpha, \\ c(1+t)^{\alpha /(\gamma-\alpha)}, & \gamma>2 \alpha,\end{cases}
$$

and the density decays pointwise to zero for any $x \in[0, y(t)) \cup$ $(y(t), a(t)]$ and $t>0$ as

$$
\begin{aligned}
& \rho(a(t), t)=\left((\gamma-\alpha) t+\rho_{0}\left(a_{0}\right)^{\alpha-\gamma}\right)^{-1 /(\gamma-\alpha)}, \\
& \rho(x, t) \leq C(1+t)^{-1 / \gamma-\alpha} \\
& +\left\{\begin{array}{r}
C(1+t)^{-(\gamma-1) /(3 \gamma+2 \alpha-1)}, \\
1<\gamma<2 \alpha, \\
C(1+t)^{-((\gamma-1) /(3 \gamma+2 \alpha-1))+\gamma}, \\
\gamma=2 \alpha, \\
C(1+t)^{-\alpha(\gamma-1) /(3 \gamma+2 \alpha-1)(\gamma-\alpha)}, \\
\gamma>2 \alpha,
\end{array}\right. \\
& +\left\{\begin{array}{c}
C(1+t)^{-2 /(3 \gamma+2 \alpha-1)}, \\
1<\gamma<2 \alpha, \\
C(1+t)^{-(2 /(3 \gamma+2 \alpha-1))+\gamma}, \\
\gamma=2 \alpha, \\
C(1+t)^{-2 \alpha /(3 \gamma+2 \alpha-1)(\gamma-\alpha)}, \\
\gamma>2 \alpha,
\end{array}\right.
\end{aligned}
$$

where $C>0$ and $c>0$ are positive constants independent of time, and $v \in(0,1)$ is a small constant.

Remark 3. Theorem 2 holds for the Saint-Venant model for shallow water; that is, $\gamma=2, \alpha=1$.

Remark 4. Fang-Zhang [10] obtained that

$$
\left|\left[\rho_{0}^{\alpha}\left(y_{0}\right)\right]\right| e^{-C_{0} t} \leq\left|\left[\rho^{\alpha}(y(t), t)\right]\right|,
$$

which show that the discontinuity in the density persists for all time. However, in this paper, from (15) we can shows that the discontinuity in the density decays at an algebraic rate in time; namely,

$$
\begin{aligned}
\left|\left[\rho^{\alpha}(y(t), t)\right]\right| \leq & C(1+t)^{-\alpha /(\gamma-\alpha)} \\
& +\left\{\begin{array}{r}
C(1+t)^{-\alpha(\gamma-1) /(3 \gamma+2 \alpha-1)}, \\
1<\gamma<2 \alpha, \\
C(1+t)^{-(\alpha(\gamma-1) /(3 \gamma+2 \alpha-1))+\alpha \nu,} \\
\gamma=2 \alpha, \\
C(1+t)^{-\alpha^{2}(\gamma-1) /(3 \gamma+2 \alpha-1)(\gamma-\alpha)}, \\
\gamma>2 \alpha,
\end{array}\right. \\
+ & \left\{\begin{array}{r}
C(1+t)^{-2 \alpha /(3 \gamma+2 \alpha-1)}, \\
1<\gamma<2 \alpha, \\
C(1+t)^{-(2 \alpha /(3 \gamma+2 \alpha-1))+\gamma,} \\
\gamma=2 \alpha, \\
C(1+t)^{-2 \alpha^{2} /(3 \gamma+2 \alpha-1)(\gamma-\alpha),} \\
\gamma>2 \alpha,
\end{array}\right.
\end{aligned}
$$

where $C>0$ is a positive constant independent of time, and $v \in(0,1)$ is a small constant. 


\section{The A Priori Estimates}

According to the analysis made in [29], there is a curve $x=$ $y(t)$ defined by

$$
\frac{d y(t)}{d t}=u(y(t), t), \quad y(0)=y_{0}, t>0
$$

along which the Rankine-Hugoniot conditions hold

$$
[u(y(t), t)]=0, \quad\left[\rho^{\gamma}(y(t), t)\right]=\left[\rho^{\alpha} u_{x}(y(t), t)\right],
$$

where $[f(y(t), t)]:=f(y(t)+0, t)-f(y(t)-0, t)$.

It is convenient to make use of the Lagrange coordinates in order to establish the uniformly a-priori estimates. Let $(\rho, u, a)$ be a piecewise regular solution to the FBVP (1) and (2), and take the Lagrange coordinates transform

$$
\xi=\int_{0}^{x} \rho(z, t) d z, \quad \tau=t
$$

Since the conservation of total mass holds

$$
\int_{0}^{a(t)} \rho(z, t) d z=\int_{0}^{a_{0}} \rho_{0}(z) d z:=1>0,
$$

the boundaries $x=0$ and $x=a(t)$ are transformed into $\xi=0$ and $\xi=1$, respectively, and the domain $[0, a(t)]$ is transformed into $[0,1]$, the curve $x=y(t)$ in the Eulerian coordinates is changed to a line $\xi=\xi_{0}$ in the Lagrangian coordinates, where

$$
\xi_{0}:=\int_{0}^{y(t)} \rho(z, t) d z=\int_{0}^{y_{0}} \rho_{0}(z) d z,
$$

and the jump conditions become

$$
\left[u\left(\xi_{0}, \tau\right)\right]=0, \quad\left[\rho^{\gamma}\left(\xi_{0}, \tau\right)\right]=\left[\rho^{1+\alpha} u_{\xi}\left(\xi_{0}, \tau\right)\right] .
$$

Meanwhile, the FBVP (1) and (2) is reformulated into

$$
\begin{gathered}
\rho_{\tau}+\rho^{2} u_{\xi}=0, \\
u_{\tau}+\left(\rho^{\gamma}\right)_{\xi}=\left(\rho^{1+\alpha} u_{\xi}\right)_{\xi} \\
\left(\rho^{\gamma}-\rho^{1+\alpha} u_{\xi}\right)(0, \tau)=\left(\rho^{\gamma}-\rho^{1+\alpha} u_{\xi}\right)(1, \tau)=0, \\
\left(\rho_{0}, u_{0}\right)=\left(\rho_{0}, u_{0}\right)(\xi), \quad \xi \in[0,1],
\end{gathered}
$$

where the initial data satisfies

$$
\begin{gathered}
\inf _{\left[0, \xi_{0}\right) \cup\left(\xi_{0}, 1\right]} \rho_{0} \geq \rho_{-}>0, \\
\left(\rho_{0}, u_{0}\right) \in W^{1, \infty}\left(\left[0, \xi_{0}\right) \cup\left(\xi_{0}, 1\right]\right), \\
\left(\rho_{0}^{\gamma}-\rho_{0}^{1+\alpha} u_{0 x}\right)(1)=0, \\
\rho_{0}\left(\xi_{0}-0\right)>\rho_{0}\left(\xi_{0}+0\right),
\end{gathered}
$$

for some constant $\rho_{-}>0$, and the consistencies between initial data and boundary value hold.

Next, we will give the a-priori estimates for the solution $(\rho, u)$ to the FBVP $(24)$. Similarly to the arguments used in $[10,16,20]$, we can establish the following a priori estimates and omit the details here.
Lemma 5. Let $T>0$. Under the assumptions of Theorem 2, it holds for any piecewise regular solution $(\rho, u)$ to the FBVP $(24)$ that

$$
\begin{gathered}
\int_{0}^{1}\left(\frac{u^{2}}{2}+\frac{1}{\gamma-1} \rho^{\gamma-1}\right) d \xi+\int_{0}^{\tau} \int_{0}^{1} \rho^{1+\alpha} u_{\xi}^{2} d \xi d s \\
=\int_{0}^{1}\left(\frac{u_{0}^{2}}{2}+\frac{1}{\gamma-1} \rho_{0}^{\gamma-1}\right) d \xi, \quad \tau \in[0, T], \\
\rho(\xi, \tau) \leq C, \quad(\xi, \tau) \in\left[0, \xi_{0}\right) \cup\left(\xi_{0}, 1\right] \times[0, T], \\
\int_{0}^{1} u^{2 n} d \xi+n(2 n-1) \int_{0}^{\tau} \int_{0}^{1} \rho^{1+\alpha} u^{2 n-2} u_{\xi}^{2} d \xi d s \\
\leq C(T), \quad \tau \in[0, T], \\
f_{0}^{1}\left(\rho^{\alpha}\right)_{\xi}^{2 n} d \xi \leq C(T), \quad \tau \in[0, T],
\end{gathered}
$$

for any positive integer $n \in N, C>0$ denotes a constant independent of time and $C(T)>0$ denotes a constant dependent on time, where $f_{0}^{1}:=\int_{0}^{\xi_{0}}+\int_{\xi_{0}}^{1}$.

Lemma 6. Let $T>0$. Under the assumptions of Theorem 2, it holds for any piecewise regular solution $(\rho, u)$ to the FBVP $(24)$ that

$$
\begin{gathered}
\rho(0, \tau)=\left((\gamma-\alpha) \tau+\rho_{0}(0)^{\alpha-\gamma}\right)^{-1 /(\gamma-\alpha)}, \\
\rho\left(\xi_{0}-0, \tau\right)>\rho\left(\xi_{0}+0, \tau\right), \quad \tau \in[0, T] .
\end{gathered}
$$

Proof. From $(24)_{1}$ and $(24)_{3}$, we have

$$
\rho_{\tau}(0, \tau)+\rho^{\gamma-\alpha+1}(0, \tau)=0,
$$

which yields (30) similarly, because of (23) and (24) $)_{1}$, it holds that

$$
\left[\rho^{\alpha}\right]_{\tau}+\alpha\left[\rho^{\gamma}\right]=0
$$

which together with (25) implies

$$
\left[\rho^{\alpha}\right]=\left[\rho_{0}^{\alpha}\right] \exp \left\{-\alpha \int_{0}^{\tau} \frac{\left[\rho^{\gamma}\right]}{\left[\rho^{\alpha}\right]} d s\right\}<0
$$

Lemma 7. Let $T>0$. Under the assumptions of Theorem 2, it holds for any piecewise regular solution $(\rho, u)$ to the FBVP $(24)$ that

$$
\begin{aligned}
& \frac{1}{2} f_{0}^{1}\left(u+\frac{\left(\rho^{\alpha}\right)_{\xi}}{\alpha}\right)^{2} d \xi+\frac{1}{\gamma-1} \int_{0}^{1} \rho^{\gamma-1} d \xi \\
& \quad+\gamma \int_{0}^{\tau} f_{0}^{1} \rho^{\gamma+\alpha-2} \rho_{\xi}^{2} d \xi d s+\rho^{\gamma}(1, \tau) a(\tau)
\end{aligned}
$$




$$
\begin{aligned}
&+\gamma \int_{0}^{\tau} \rho^{2 \gamma-\alpha}(1, s) a(s) d s-\left[\rho^{\gamma}\right] y(\tau) \\
&-\gamma \int_{0}^{\tau}\left[\rho^{\gamma+1}\right] u_{\xi} y(s) d s \\
&=\frac{1}{2} f_{0}^{1}\left(u_{0}+\frac{\left(\rho^{\alpha}\right)_{\xi}}{\alpha}\right)^{2} d \xi \\
&+\frac{1}{\gamma-1} \int_{0}^{1} \rho_{0}^{\gamma-1} d \xi+\rho_{0}^{\gamma}(1) a_{0}-\left[\rho_{0}^{\gamma}\right] y_{0} \\
& \tau \in[0, T],
\end{aligned}
$$

where $a(\tau)$ satisfies $a^{\prime}(\tau)=u(0, \tau)$ and $a(0)=a_{0}, y(\tau)$ satisfies $y^{\prime}(\tau)=u\left(\xi_{0}, \tau\right)$ and $y(0)=y_{0}$.

Proof. Multiplying (24) ${ }_{1}$ by $\rho^{\alpha-1}$ gives

$$
\frac{\left(\rho^{\alpha}\right)_{\tau}}{\alpha}+\rho^{1+\alpha} u_{\xi}=0
$$

which leads to

$$
\frac{\left(\rho^{\alpha}\right)_{\tau \xi}}{\alpha}+\left(\rho^{1+\alpha} u_{\xi}\right)_{\xi}=0
$$

Summing $(24)_{2}$ and (37), we have

$$
\left(u+\frac{\left(\rho^{\alpha}\right)_{\xi}}{\alpha}\right)_{\tau}+\left(\rho^{\gamma}\right)_{\xi}=0 .
$$

Multiplying (38) by $\left(u+\left(\rho^{\alpha}\right) \_\xi / \alpha\right)$ and integrating the result over $[0,1] \times[0, \tau]$, we get

$$
\begin{aligned}
\frac{1}{2} f_{0}^{1} & \left(u+\frac{\left(\rho^{\alpha}\right)_{\xi}}{\alpha}\right)^{2} d \xi+\frac{1}{\gamma-1} \int_{0}^{1} \rho^{\gamma-1} d \xi \\
& +\gamma \int_{0}^{\tau} f_{0}^{1} \rho^{\gamma+\alpha-2} \rho_{\xi}^{2} d \xi d s+\left.\int_{0}^{\tau} \rho^{\gamma} u\right|_{\xi=0} ^{\xi=\xi_{0}-0} d s \\
& +\left.\int_{0}^{\tau} \rho^{\gamma} u\right|_{\xi=\xi_{0}+0} ^{\xi=1} d s \\
= & \frac{1}{2} f_{0}^{1}\left(u_{0}+\frac{\left(\rho_{0}^{\alpha}\right)_{\xi}}{\alpha}\right)^{2} d \xi+\frac{1}{\gamma-1} \int_{0}^{1} \rho_{0}^{\gamma-1} d \xi,
\end{aligned}
$$

which together with the fact that

$$
\begin{aligned}
\int_{0}^{\tau} & \left.\rho^{\gamma} u\right|_{\xi=0} ^{\xi=\xi_{0}-0} d s \\
= & \int_{0}^{\tau} \rho^{\gamma}\left(\xi_{0}-0, s\right) y^{\prime}(s) d s \\
= & \left.\rho^{\gamma}\left(\xi_{0}-0, s\right) y(s)\right|_{0} ^{\tau} \\
& +\gamma \int_{0}^{\tau} \rho^{\gamma-1} \rho^{2}\left(\xi_{0}-0, s\right) u_{\xi}\left(\xi_{0}, s\right) y(s) d s \\
= & \rho^{\gamma}\left(\xi_{0}-0, \tau\right) y(\tau)-\rho_{0}^{\gamma}\left(\xi_{0}-0\right) y_{0} \\
& +\gamma \int_{0}^{\tau} \rho^{\gamma+1}\left(\xi_{0}-0, s\right) u_{\xi}\left(\xi_{0}, s\right) y(s) d s,
\end{aligned}
$$

$$
\begin{aligned}
\left.\int_{0}^{\tau} \rho^{\gamma} u\right|_{\xi=\xi_{0}+0} ^{\xi=1} d s & \\
= & \int_{0}^{\tau} \rho^{\gamma}(1, \tau) a^{\prime}(s) d s \\
& -\int_{0}^{\tau} \rho^{\gamma}\left(\xi_{0}+0, \tau\right) y^{\prime}(s) d s \\
= & \left.\rho^{\gamma}(1, s) a(s)\right|_{0} ^{\tau} \\
& +\gamma \int_{0}^{\tau} \rho^{2 \gamma-\alpha}(1, s) a(s) d s \\
& -\left.\rho^{\gamma}\left(\xi_{0}+0, s\right) y(s)\right|_{0} ^{\tau} \\
& -\gamma \int_{0}^{\tau} \rho^{\gamma+1}\left(\xi_{0}+0, s\right) u_{\xi}\left(\xi_{0}, s\right) y(s) d s \\
= & \rho^{\gamma}(1, \tau) a(\tau)-\rho_{0}^{\gamma}(1) a_{0} \\
& +\gamma \int_{0}^{\tau} b(s) \rho^{2 \gamma-\alpha}(1, s) d s \\
& -\rho^{\gamma}\left(\xi_{0}+0, \tau\right) y(\tau)+\rho_{0}^{\gamma}\left(\xi_{0}+0\right) y_{0} \\
& -\gamma \int_{0}^{\tau} \rho^{\gamma+1}\left(\xi_{0}+0, s\right) u_{\xi}\left(\xi_{0}, s\right) y(s) d s, \\
& \quad\left[\rho\left(\xi_{0}, \tau\right)\right]<0, \\
& \quad u_{\xi}\left(\xi_{0}, \tau\right)=\frac{\left[\rho^{\gamma}\right]}{\left[\rho^{1+\alpha}\right]}>0, \\
& a(\tau)=\int_{0}^{1} \frac{1}{\rho(\zeta, \tau)} d \zeta>0, \\
\xi_{0} & \frac{1}{\rho(\zeta, \tau)} d \zeta>0, \\
&
\end{aligned}
$$

gives rise to (35).

Remark 8. The estimate (35) can be written in the following form in the Eulerian coordinates; that is to say, for all $t \in$ $[0, T]$,

$$
\begin{aligned}
\frac{1}{2} f_{0}^{a(t)} & \rho\left(u+\rho^{-1}\left(\rho^{\alpha}\right)_{x}\right)^{2} d x+\frac{1}{\gamma-1} \int_{0}^{a(t)} \rho^{\gamma} d x \\
& +\gamma \int_{0}^{t} f_{0}^{a(t)} \rho^{\gamma+\alpha-3} \rho_{x}^{2} d x d s+\rho^{\gamma}(a(t), t) a(t) \\
& +\gamma \int_{0}^{t} \rho^{2 \gamma-\alpha}(a(s), s) a(s) d s \\
& -\left[\rho^{\gamma}\right] y(t)-\gamma \int_{0}^{t}\left[\rho^{\gamma}\right] u_{x} y(s) d s \\
= & \frac{1}{2} f_{0}^{a_{0}} \rho_{0}\left(u_{0}+\rho_{0}^{-1}\left(\rho_{0}^{\alpha}\right)_{x}\right)^{2} d x \\
& +\frac{1}{\gamma-1} \int_{0}^{a_{0}} \rho_{0}^{\gamma} d x+\rho_{0}^{\gamma}\left(a_{0}\right) a_{0}-\left[\rho_{0}^{\gamma}\right] y_{0} .
\end{aligned}
$$

Lemma 9. Let $T>0$, for $n \in N$, and $n>(1+\alpha) / 4(\gamma-\alpha)$. Under the assumptions of Theorem 2, it holds for any piecewise regular solution $(\rho, u)$ to the FBVP (24) that

$$
\int_{0}^{\tau}\left\|\left(\rho^{\gamma}\right)_{\xi}^{2 n}\right\|_{L^{\infty}\left(\left[0, \xi_{0}\right) \cup\left(\xi_{0}, 1\right]\right)} d s \leq C(T), \quad \tau \in[0, T] .
$$


Proof. It follows from $(24)_{1,2}$ that

$$
\begin{aligned}
\left(\rho^{\alpha}\right)_{\xi}(\xi, \tau)= & \left(\rho_{0}^{\alpha}\right)_{\xi}(\xi)-\alpha u(\xi, \tau) \\
& +\alpha u_{0}(\xi)-\alpha \int_{0}^{\tau}\left(\rho^{\gamma}\right)_{\xi}(\xi, s) d s .
\end{aligned}
$$

By means of (25), (26), and (44), we have

$$
\begin{aligned}
\int_{0}^{\tau} & \left\|\left(\rho^{\gamma}\right)_{\xi}^{2 n}\right\|_{L^{\infty}\left(\left[0, \xi_{0}\right) \cup\left(\xi_{0}, 1\right]\right)} d s \\
= & \frac{\gamma^{2 n}}{\alpha^{2 n}} \int_{0}^{\tau}\left\|\rho^{2 n(\gamma-\alpha)}\left(\rho^{\alpha}\right)_{\xi}^{2 n}\right\|_{L^{\infty}\left(\left[0, \xi_{0}\right) \cup\left(\xi_{0}, 1\right]\right)} d s \\
\leq & C(T)+C(T) \int_{0}^{\tau}\left\|\rho^{2 n(\gamma-\alpha)} u^{2 n}\right\|_{L^{\infty}\left(\left[0, \xi_{0}\right) \cup\left(\xi_{0}, 1\right]\right)} d s \\
& +C(T) \int_{0}^{\tau} \int_{0}^{s}\left\|\left(\rho^{\gamma}\right)_{\xi}^{2 n}\right\|_{L^{\infty}\left(\left[0, \xi_{0}\right) \cup\left(\xi_{0}, 1\right]\right)} d l d s \\
\leq & C(T)+C(T) \int_{0}^{\tau} \int_{0}^{s}\left\|\left(\rho^{\gamma}\right)_{\xi}^{2 n}\right\|_{L^{\infty}\left(\left[0, \xi_{0}\right) \cup\left(\xi_{0}, 1\right]\right)} d l d s,
\end{aligned}
$$

where we have used

$$
\begin{aligned}
\int_{0}^{\tau} & \left\|\rho^{2 n(\gamma-\alpha)} u^{2 n}\right\|_{L^{\infty}\left(\left[0, \xi_{0}\right) \cup\left(\xi_{0}, 1\right]\right)} d s \\
\leq & \int_{0}^{\tau} f_{0}^{1} \rho^{2 n(\gamma-\alpha)} u^{2 n} d \xi d s \\
& +\int_{0}^{\tau} f_{0}^{1}\left|\left(\rho^{2 n(\gamma-\alpha)} u^{2 n}\right)_{\xi}\right| d \xi d s \\
\leq & C(T) \quad+\rho^{4 n(\gamma-\alpha)-(1+\alpha)} u^{2 n} \\
& +C \int_{0}^{\tau} f_{0}^{1}\left(\rho^{2(2 n(\gamma-\alpha)-\alpha)} \rho^{2 \alpha-2} \rho_{\xi}^{2}+u^{4 n}\right. \\
\leq & \left.\quad+\rho^{1+\alpha} u^{2 n-2} u_{\xi}^{2}\right) d \xi d s
\end{aligned}
$$

which can be deduced from (26) and (27). Making use of Gronwall's inequality to (46), we obtain (43).

Lemma 10. Let $T>0$. Under the assumptions of Theorem 2, it holds for any piecewise regular solution $(\rho, u)$ to the FBVP (24) that

$$
\rho(\xi, \tau) \geq C(T), \quad(\xi, \tau) \in\left[0, \xi_{0}\right) \cup\left(\xi_{0}, 1\right] \times[0, T] .
$$

Proof. Denote

$$
v(\xi, \tau)=\frac{1}{\rho(\xi, \tau)} .
$$

By $(24)_{1}$, we have

$$
v_{\tau}=u_{\xi}
$$

Multiplying (49) by $\beta v^{\beta-1}$, integrating the result over $[0,1] \times$ $[0, \tau]$, and using $(43),(44)$, we can obtain that for $\beta \in(1,2)$

$$
\begin{gathered}
\int_{0}^{1} v^{\beta} d \xi+\beta(\beta-1) \int_{0}^{\tau} \int_{0}^{1} v^{\alpha+\beta-1} u^{2} d \xi d s \\
=\int_{0}^{1} v_{0}^{\beta} d \xi+\left.\beta \int_{0}^{\tau} v^{\beta-1} u d s\right|_{\xi=0} ^{\xi=\xi_{0}-0}
\end{gathered}
$$

$$
\begin{aligned}
& +\left.\beta \int_{0}^{\tau} v^{\beta-1} u d s\right|_{\xi_{0}+0} ^{\xi=1}+\frac{\beta(\beta-1)}{\alpha} \int_{0}^{\tau} f_{0}^{1} v^{\alpha+\beta-1} u\left(\rho_{0}^{\alpha}\right)_{\xi} d \xi d s \\
& +\beta(\beta-1) \int_{0}^{\tau} \int_{0}^{1} v^{\alpha+\beta-1} u u_{0} d \xi d s-\beta(\beta-1) \\
& \times \int_{0}^{\tau} \int_{0}^{1} v^{\alpha+\beta-1} u \int_{0}^{s}\left(\rho^{\gamma}\right)_{\xi} d l d \xi d s \\
& \leq C(T)+C \int_{0}^{\tau} v^{\beta-1} u(1, s) d s+\frac{\beta(\beta-1)}{2} \\
& \times \int_{0}^{\tau} \int_{0}^{1} v^{\alpha+\beta-1} u^{2} d \xi d s+C(T) \int_{0}^{\tau} \int_{0}^{1} v^{\beta} d \xi d s,
\end{aligned}
$$

where we use the fact that

$$
\begin{aligned}
& \int_{0}^{\tau} v^{\beta-1}\left(\xi_{0}-0, s\right) u\left(\xi_{0}, s\right) d s \\
& \quad-\int_{0}^{\tau} v^{\beta-1}\left(\xi_{0}+0, s\right) u\left(\xi_{0}, s\right) d s \\
&=\int_{0}^{\tau} \rho^{1-\beta}\left(\xi_{0}-0, s\right) y^{\prime}(s) d s \\
& \quad-\int_{0}^{\tau} \rho^{1-\beta}\left(\xi_{0}+0, s\right) y^{\prime}(s) d s \\
&=\left.\quad \rho^{1-\beta}\left(\xi_{0}-0, s\right) y(s)\right|_{0} ^{\tau}-\left.\rho^{1-\beta}\left(\xi_{0}+0, s\right) y(s)\right|_{0} ^{\tau} \\
& \quad+(1-\beta) \int_{0}^{\tau} \rho^{2-\beta}\left(\xi_{0}-0, s\right) u_{\xi}\left(\xi_{0}, s\right) y(s) d s \\
& \quad-(1-\beta) \int_{0}^{\tau} \rho^{2-\beta}\left(\xi_{0}+0, s\right) u_{\xi}\left(\xi_{0}, s\right) y(s) d s \\
&=-\left[\rho^{1-\beta}\right] y(\tau)+\left[\rho_{0}^{1-\beta}\right] y_{0} \\
& \quad+(\beta-1) \int_{0}^{\tau}\left[\rho^{2-\beta}\right] u_{\xi}\left(\xi_{0}, s\right) y(s) d s \\
& \leq {\left[\rho_{0}^{1-\beta}\right] y_{0} \leq C . }
\end{aligned}
$$

Since it holds that

$$
\rho(1, \tau)=\left((\gamma-\alpha) \tau+\rho_{0}(1)^{\alpha-\gamma}\right)^{-1 /(\gamma-\alpha)} \geq C(T),
$$

we have from (25) that

$$
\int_{0}^{\tau} v^{\beta-1} u(1, s) d \xi
$$

$$
\begin{aligned}
& \leq C(T) \int_{0}^{\tau}\left(\left(\int_{0}^{1} u^{2} d \xi\right)^{1 / 2}\right. \\
& \left.\quad+\left(\int_{0}^{1} \rho^{1+\alpha} u_{\xi}^{2} d \xi\right)^{1 / 2}\left(\int_{0}^{1} v^{1+\alpha} d \xi\right)^{1 / 2}\right) d s \\
& \leq C(T)+C(T) \int_{0}^{\tau} \int_{0}^{1} v^{\beta} d \xi d s .
\end{aligned}
$$


Substituting (53) in (50), we have

$$
\begin{gathered}
\int_{0}^{1} v^{\beta} d \xi+\frac{\beta(\beta-1)}{2} \int_{0}^{\tau} \int_{0}^{1} v^{\alpha+\beta-1} u^{2} d \xi d s \\
\leq C(T)+C(T) \int_{0}^{\tau} \int_{0}^{1} v^{\beta} d \xi d s .
\end{gathered}
$$

Using Gronwall's inequality, we get from (54) that

$$
\int_{0}^{1} v^{\beta} d \xi \leq C(T) .
$$

It follows from (29) and (55) that

$$
\begin{aligned}
v^{\beta}(\xi, \tau) \leq & \int_{0}^{1} v^{\beta} d \xi+f_{0}^{1}\left|v_{\xi}^{\beta}\right| d \xi \\
\leq & C(T)+C\left(f_{0}^{1} \rho^{-\beta-1}\left|\rho_{\xi}\right| d \xi\right) \\
\leq & C(T)+C\left(\int_{0}^{1} \rho^{-2 n(\alpha+\beta) /(2 n-1)} d \xi\right)^{(2 n-1) / 2 n} \\
& \times\left(f_{0}^{1}\left(\rho^{\alpha}\right)_{\xi}^{2 n} d \xi\right)^{1 / 2 n} \\
\leq & C(T)+C(T) \sup _{\left[0, \xi_{0}\right) \cup\left(\xi_{0}, 1\right]} v^{\alpha+\beta / 2 n}\left(\int_{0}^{1} v^{\beta} d \xi\right)^{(2 n-1) / 2 n} \\
\leq & C(T)+C(T) \sup _{\left[0, \xi_{0}\right) \cup\left(\xi_{0}, 1\right]}\left(v^{\beta}(\xi, \tau)\right)^{\alpha / \beta+1 / 2 n},
\end{aligned}
$$

as $\alpha \in(0,1], \beta \in(1,2)$; for some $n \in N$ large enough, we have

$$
\frac{\alpha}{\beta}+\frac{1}{2 n}<1
$$

which implies (47).

We also have the regularity estimates for the solution $(\rho, u)$ to the $\operatorname{FBVP}(24)$ as follows.

Lemma 11. Let $T>0$. Under the assumptions of Theorem 2, it holds for any piecewise regular solution $(\rho, u)$ to the FBVP (24) that

$$
\begin{gathered}
\rho \in L^{\infty}\left(0, T ; H^{1}\left(\left[0, \xi_{0}\right) \cup\left(\xi_{0}, 1\right]\right)\right) \\
\cap C^{0}\left(\left[0, \xi_{0}\right) \cup\left(\xi_{0}, 1\right] \times[0, T]\right), \\
u \in L^{\infty}\left(0, T ; H^{1}([0,1])\right) \cap L^{2}\left(0, T ; H^{2}([0,1])\right), \\
u_{\tau} \in L^{2}\left(0, T ; L^{2}([0,1])\right), \quad a(\tau) \in H^{1}([0, T]), \\
\left(\rho^{\gamma}-\rho^{1+\alpha} u_{\xi}\right) \in L^{\infty}\left(0, T ; L^{2}\left(\left[0, \xi_{0}\right) \cup\left(\xi_{0}, 1\right]\right)\right) .
\end{gathered}
$$

If it is also satisfied that

$$
u_{0} \in H^{2}([0,1])
$$

then the piecewise regular solution $(\rho, u)$ has the regularities

$$
\begin{gathered}
\rho \in L^{\infty}\left(0, T ; H^{1}\left(\left[0, \xi_{0}\right) \cup\left(\xi_{0}, 1\right]\right)\right), \\
\rho_{\tau} \in L^{\infty}\left(0, T ; L^{2}\left(\left[0, \xi_{0}\right) \cup\left(\xi_{0}, 1\right]\right)\right), \\
u \in L^{\infty}\left(0, T ; H^{1}([0,1])\right) \cap L^{2}\left(0, T ; H^{2}([0,1])\right), \\
u_{\tau} \in L^{\infty}\left(0, T ; L^{2}([0,1])\right) \cap L^{2}\left(0, T ; H^{1}([0,1])\right), \\
a(\tau) \in H^{2}([0, T]), \\
\left(\rho^{\gamma}-\rho^{1+\alpha} u_{\xi}\right) \in L^{\infty}\left(0, T ; H^{1}\left(\left[0, \xi_{0}\right) \cup\left(\xi_{0}, 1\right]\right)\right) .
\end{gathered}
$$

Proof. Multiplying $(24)_{2}$ by $u_{\tau}$, integrating the result over $[0,1]$, and making use of the boundary condition $(24)_{3}$, after a direct computation and recombination, we deduce

$$
\begin{aligned}
& \frac{1}{2} \int_{0}^{1} \rho^{1+\alpha} u_{\xi}^{2} d \xi+\int_{0}^{\tau} \int_{0}^{1} u_{s}^{2} d \xi d s \\
& =-\int_{0}^{1} \rho_{0}^{\gamma} u_{0 \xi} d \xi+\frac{1}{2} \int_{0}^{1} \rho^{1+\alpha} u_{0 \xi}^{2} d \xi+\int_{0}^{1} \rho^{\gamma} u_{\xi} d \xi \\
& \quad+\gamma \int_{0}^{\tau} \int_{0}^{1} \rho^{1+\gamma} u_{\xi}^{2} d \xi d s-\frac{1+\alpha}{2} \int_{0}^{\tau} \int_{0}^{1} \rho^{2+\alpha} u_{\xi}^{3} d \xi d s \\
& \leq C+\frac{1}{4} \int_{0}^{1} \rho^{1+\alpha} u_{\xi}^{2} d \xi+\int_{0}^{\tau} \int_{0}^{1}\left|u_{\xi}\right|^{3} d \xi d s,
\end{aligned}
$$

where we have used (26) and (27). On the other hand, integrating $(24)_{2}$ over $[\xi, 1]$ and making use of $(23)$ and the boundary conditions $(24)_{3}$, it holds that

$$
\rho^{1+\alpha} u_{\xi}=\rho^{\gamma}-\int_{\xi}^{1} u_{\tau} d \xi \leq C+C\left(\int_{0}^{1} u_{\tau}^{2} d \xi\right)^{1 / 2},
$$

which implies

$$
\sup _{\left[0, \xi_{0}\right) \cup\left(\xi_{0}, 1\right]} u_{\xi} \leq C(T)+C(T)\left(\int_{0}^{1} u_{\tau}^{2} d \xi\right)^{1 / 2} .
$$

It holds from (61) and (63) that

$$
\begin{aligned}
& \frac{1}{2} \int_{0}^{1} u_{\xi}^{2} d \xi+\int_{0}^{\tau} \int_{0}^{1} u_{s}^{2} d \xi d s \\
& \quad \leq C(T)+C(T) \int_{0}^{\tau} \sup _{\left[0, \xi_{0}\right) \cup\left(\xi_{0}, 1\right]}\left|u_{\xi}\right| \int_{0}^{1} u_{\xi}^{2} d \xi d s \\
& \quad \leq C(T)+C(T) \int_{0}^{\tau}\left(1+\int_{0}^{1} u_{\tau}^{2} d \xi\right)^{1 / 2} \int_{0}^{1} u_{\xi}^{2} d \xi d s \\
& \quad \leq \frac{1}{2} \int_{0}^{\tau} \int_{0}^{1} u_{s}^{2} d \xi d s+\int_{0}^{\tau}\left\|u_{\xi}\right\|_{L^{2}}^{2} \int_{0}^{1} u_{\xi}^{2} d \xi d s ;
\end{aligned}
$$

using Gronwall's inequality, (26), and (47), we have

$$
\int_{0}^{1} u_{\xi}^{2} d \xi+\int_{0}^{\tau} \int_{0}^{1} u_{s}^{2} d \xi d s \leq C(T),
$$

where $C(T)>0$ denotes a constant dependent of time. 
Differentiating $(24)_{2}$ with respect to $\tau$, we get

$$
u_{\tau \tau}+(\rho)_{\xi \tau}^{\gamma}=\left(\rho^{1+\alpha} u_{\xi}\right)_{\xi \tau} .
$$

Taking inner product between (66) and $u_{\tau}$, integrating the results over $[0,1]$, and using the boundary conditions $(24)_{3}$, it holds that

$$
\frac{1}{2} \frac{d}{d \tau} \int_{0}^{1} u_{\tau}^{2} d \xi=\int_{0}^{1}\left(\rho^{\gamma}\right)_{\tau} u_{\xi \tau} d \xi-\int_{0}^{1}\left(\rho^{1+\alpha} u_{\xi}\right)_{\tau} u_{\xi \tau} d \xi
$$

The terms on the right-hand side of (67) can be bounded, respectively,as described below:

$$
\begin{aligned}
\int_{0}^{1}\left(\rho^{\gamma}\right)_{\tau} u_{\xi \tau} d \xi \\
=-\int_{0}^{1} \gamma \rho^{\gamma+1} u_{\xi} u_{\xi \tau} d \xi \\
\leq-\frac{\gamma}{2} \frac{d}{d \tau} \int_{0}^{1} \rho^{\gamma+1} u_{\xi}^{2} d \xi \\
\quad+C \int_{0}^{1}\left(\rho^{1+\alpha} u_{\xi}^{2}+\rho^{2 \gamma-\alpha+3} u_{\xi}^{4}\right) d \xi \\
\quad-\int_{0}^{1}\left(\rho^{1+\alpha} u_{\xi}\right)_{\tau} u_{\xi \tau} d \xi \\
=-\int_{0}^{1}\left((1+\alpha) \rho^{\alpha} \rho_{\tau} u_{\xi}+\rho^{1+\alpha} u_{\xi \tau}\right) u_{\xi \tau} d \xi \\
\leq-\frac{1}{2} \int_{0}^{1} \rho^{1+\alpha} u_{\xi \tau}^{2} d \xi+C \int_{0}^{1} \rho^{3+\alpha} u_{\xi}^{4} d \xi .
\end{aligned}
$$

Summing (67) and (68) together and making use of (27) and (65), we obtain

$$
\begin{gathered}
\frac{1}{2} \frac{d}{d \tau} \int_{0}^{1} u_{\tau}^{2} d \xi+\frac{\gamma}{2} \frac{d}{d \tau} \int_{0}^{1} \rho^{\gamma+1} u_{\xi}^{2} d \xi+\frac{1}{2} \int_{0}^{1} \rho^{1+\alpha} u_{\xi \tau}^{2} d \xi \\
\leq C \int_{0}^{1}\left(\rho^{1+\alpha} u_{\xi}^{2}+\rho^{2 \gamma-\alpha+3} u_{\xi}^{4}\right) d \xi+\int_{0}^{1} \rho^{3+\alpha} u_{\xi}^{4} d \xi \\
\leq C(T)+C\left\|\rho^{1+\alpha} u_{\xi}\right\|_{L^{\infty}([0,1])}^{2} \int_{0}^{1} \rho^{1-\alpha} u_{\xi}^{2} d \xi .
\end{gathered}
$$

Substituting (62) into (69), it follows from (27), (47), and (59) that

$$
\begin{gathered}
\frac{1}{2} \int_{0}^{1} u_{\tau}^{2} d \xi+\frac{\gamma}{2} \int_{0}^{1} \rho^{\gamma+1} u_{\xi}^{2} d \xi+\frac{1}{2} \int_{0}^{\tau} \int_{0}^{1} \rho^{1+\alpha} u_{\xi \tau}^{2} d \xi d s \\
\leq C(T)+C(T) \int_{0}^{\tau} \int_{0}^{1} u_{s}^{2} d \xi d s,
\end{gathered}
$$

which together with Gronwall's inequality, (27), (47), and (65) yields

$$
\int_{0}^{1} u_{\xi}^{2} d \xi+\int_{0}^{1} u_{\tau}^{2} d \xi+\int_{0}^{\tau} \int_{0}^{1} u_{s}^{2} d \xi d s+\int_{0}^{\tau} \int_{0}^{1} u_{\xi s}^{2} d \xi d s \leq C(T)
$$

which implies $\left(\rho^{\gamma}-\rho^{1+\alpha} u_{\xi}\right) \in L^{\infty}\left(0, T ; H^{1}([0,1])\right)$, and it follows from the definition of $a^{\prime}(\tau)=u(0, \tau)$ that $a(\tau) \in$ $H^{2}([0, T])$. The proof of this Lemma is completed.
Lemma 12. Let $T>0$. Under the assumptions of Theorem 2, it holds for any piecewise regular solution $(\rho, u)$ to the FBVP $(24)$ that

$$
\left|\left[\rho_{0}^{\alpha}\left(\xi_{0}\right)\right]\right| e^{-C_{0} \tau} \leq\left|\left[\rho^{\alpha}\left(\xi_{0}, \tau\right)\right]\right|, \quad \tau \in[0, T],
$$

where $C_{0}$ is a positive constant independent of time.

Proof. From (27) and (34), we can obtain (72).

Finally, we will give the large time behaviors of the interface and decay rate of the density as follows.

Lemma 13. Let $(\rho, u, a)$ be any piecewise regular solution to the FBVP (1) and (2). Under the assumptions of Theorem 2, it holds for $\alpha \in(0,1]$ and time $t>0$ large enough that

$$
C(1+t)^{\gamma /(\gamma-\alpha)} \geq a(t) \geq \begin{cases}c(1+t), & 1<\gamma<2 \alpha \\ c(1+t)^{1-\gamma}, & \gamma=2 \alpha \\ c(1+t)^{\alpha /(\gamma-\alpha)}, & \gamma>2 \alpha\end{cases}
$$

and the density decays pointwise to zero for any $x \in[0, a(t)]$ and $t>0$ as

$$
\begin{aligned}
& \rho(a(t), t)=\left((\gamma-\alpha) t+\rho_{0}\left(a_{0}\right)^{\alpha-\gamma}\right)^{-1 /(\gamma-\alpha)} \\
& \rho(x, t) \leq C(1+t)^{-1 /(\gamma-\alpha)} \\
& +\left\{\begin{array}{c}
C(1+t)^{-(\gamma-1) /(3 \gamma+2 \alpha-1)}, \\
1<\gamma<2 \alpha, \\
C(1+t)^{-((\gamma-1) /(3 \gamma+2 \alpha-1))+\nu,} \\
\gamma=2 \alpha, \\
C(1+t)^{-\alpha(\gamma-1) /(3 \gamma+2 \alpha-1)(\gamma-\alpha)}, \\
\gamma>2 \alpha,
\end{array}\right. \\
& +\left\{\begin{array}{c}
C(1+t)^{-2 /(3 \gamma+2 \alpha-1)}, \\
1<\gamma<2 \alpha, \\
C(1+t)^{-(2 /(3 \gamma+2 \alpha-1))+\nu}, \\
\gamma=2 \alpha, \\
C(1+t)^{-2 \alpha /(3 \gamma+2 \alpha-1)(\gamma-\alpha)}, \\
\gamma>2 \alpha,
\end{array}\right.
\end{aligned}
$$

where $C>0$ and $c>0$ are positive constants independent of time and $v \in(0,1)$ is a small constant.

Proof. We introduce the following functional $H(t)$ in the Eulerian form as [22, 28]:

$H(t)$

$$
\begin{aligned}
= & \int_{0}^{a(t)}(x-(1+t) u(x, t))^{2} \rho(x, t) d x \\
& +\frac{2}{\gamma-1}(1+t)^{2} \int_{0}^{a(t)} \rho^{\gamma}(x, t) d x \\
= & \int_{0}^{a(t)} x^{2} \rho(x, t) d x-2(1+t)
\end{aligned}
$$




$$
\begin{aligned}
& \times \int_{0}^{a(t)} x \rho u d x+(1+t)^{2} \int_{0}^{a(t)}\left(\rho u^{2}+\frac{2}{\gamma-1} \rho^{\gamma}\right) d x \\
:= & I_{1}+I_{2}+I_{3} .
\end{aligned}
$$

Differentiating (76) with respect to $t$, using (1), (2), and $a^{\prime}(t)=$ $u(a(t), t)$, we have

$$
\begin{aligned}
I_{1}^{\prime} & =\int_{0}^{a(t)} x^{2} \rho_{t} d x+a^{2}(t) \rho(a(t), t) a^{\prime}(t) \\
& =2 \int_{0}^{a(t)} x \rho u d x+y^{2}(t) y^{\prime}(t)[\rho], \\
I_{2}^{\prime}= & -2 \int_{0}^{a(t)} x \rho u d x-2(1+t) \\
& \times \int_{0}^{a(t)}\left(\rho u^{2}+\rho^{\gamma}\right) d x+2(1+t) \int_{0}^{a(t)} \rho^{\alpha} u_{x} d x \\
& -2(1+t) y(t)\left(y^{\prime}(t)\right)^{2}[\rho], \\
I_{3}^{\prime}= & 2(1+t) \int_{0}^{a(t)}\left(\rho u^{2}+\frac{2}{\gamma-1} \rho^{\gamma}\right) d x \\
& -2(1+t)^{2} \int_{0}^{a(t)} \rho^{\alpha} u_{x}^{2} d x \\
& +(1+t)^{2}\left(y^{\prime}(t)\right)^{3}[\rho]+\frac{2}{\gamma-1}(1+t)^{2} y^{\prime}(t)\left[\rho^{\gamma}\right] .
\end{aligned}
$$

Combining (77), we deduce

$$
\begin{aligned}
H^{\prime}(t)= & \frac{2(3-\gamma)}{\gamma-1}(1+t) \int_{0}^{a(t)} \rho^{\gamma} d x+2(1+t) \\
& \times \int_{0}^{a(t)} \rho^{\alpha} u_{x} d x-2(1+t)^{2} \int_{0}^{a(t)} \rho^{\alpha} u_{x}^{2} d x \\
& +\left(y(t)-(1+t) y^{\prime}(t)\right)^{2} y^{\prime}(t)[\rho] \\
& +\frac{2}{\gamma-1}(1+t)^{2} y^{\prime}(t)\left[\rho^{\gamma}\right] \\
\leq & \frac{2(3-\gamma)}{\gamma-1}(1+t) \int_{0}^{a(t)} \rho^{\gamma} d x+\frac{1}{2} \int_{0}^{a(t)} \rho^{\alpha} d x,
\end{aligned}
$$

where we use the fact that as $t>0$ becomes large enough; it holds from (31) and

$$
\begin{aligned}
y(t) & =\int_{0}^{\xi_{0}} \frac{1}{\rho(\zeta, t)} d \zeta \\
& =\int_{0}^{t} u(y(s), s) d s+y_{0}>0, \quad t \in[0,+\infty)
\end{aligned}
$$

that

$$
y^{\prime}(t)=u(y(t), t) \geq 0 .
$$

If $\gamma \geq 3$, we have from (78) and the conservation of mass that

$$
\begin{aligned}
H^{\prime}(t) & \leq \int_{0}^{a(t)} \rho^{\alpha} d x \\
& \leq\left(\int_{0}^{a(t)} \rho d x\right)^{\alpha}\left(\int_{0}^{a(t)} 1 d x\right)^{1-\alpha} \\
& \leq C a(t)^{1-\alpha} .
\end{aligned}
$$

Hence, it holds that

$$
\begin{gathered}
H(t) \leq H(0)+C \int_{0}^{t} a(s)^{1-\alpha} d s \leq C\left(1+\int_{0}^{t} a(s)^{1-\alpha} d s\right), \\
\int_{0}^{a(t)} \rho^{\gamma} d x \leq C\left(1+\int_{0}^{t} a(s)^{1-\alpha} d s\right)(1+t)^{-2} .
\end{gathered}
$$

From (30) and (35), we obtain

$$
a(t) \leq C \rho^{-\gamma}(a(t), t) \leq C(1+t)^{\gamma /(\gamma-\alpha)},
$$

and then

$$
\int_{0}^{t} a(s)^{1-\alpha} d s \leq C(1+t)^{\gamma(1-\alpha) /(\gamma-\alpha)+1},
$$

which with (83) implies

$$
\int_{0}^{a(t)} \rho^{\gamma} d x \leq C(1+t)^{-\alpha(\gamma-1) /(\gamma-\alpha)}, \quad \gamma \geq 3 .
$$

If $1<\gamma<3$, we deduce from (76), (78), and the conservation of mass that

$$
\begin{aligned}
H^{\prime}(t) & =(3-\gamma)(1+t)^{-1} H(t)+2 \int_{0}^{a(t)} \rho^{\alpha} d x \\
& \leq(3-\gamma)(1+t)^{-1} H(t)+C a(t)^{1-\alpha},
\end{aligned}
$$

to which the application of Gronwall's inequality gives

$$
\begin{gathered}
H(t) \leq C\left(H(0)+\int_{0}^{t} a(s)^{1-\alpha}(1+s)^{\gamma-3} d s\right)(1+t)^{3-\gamma}, \\
\int_{0}^{a(t)} \rho^{\gamma} d x \leq C\left(1+\int_{0}^{t} a(s)^{1-\alpha}(1+s)^{\gamma-3} d s\right)(1+t)^{1-\gamma} .
\end{gathered}
$$

We get from (84) that

$$
\begin{aligned}
& \int_{0}^{t} a(s)^{1-\alpha}(1+s)^{\gamma-3} d s \\
& \quad \leq \begin{cases}C(1+t)^{\gamma(1-\alpha) /(\gamma-\alpha)+\gamma-2}, & \gamma \in(1,3), \gamma \neq 2 \alpha, \\
C \ln (1+t), & \gamma=2 \alpha,\end{cases}
\end{aligned}
$$

which together with (89) yields

$$
\int_{0}^{a(t)} \rho^{\gamma} d x \leq \begin{cases}C(1+t)^{-(\gamma-1)}, & \gamma \in(1,2 \alpha), \\ C(1+t)^{-(\gamma-1)} \ln (1+t), & \gamma=2 \alpha, \\ C(1+t)^{-\alpha(\gamma-1) /(\gamma-\alpha)}, & \gamma \in(2 \alpha, 3) .\end{cases}
$$


Also, it follows from the conservation of mass and Hölder's inequality that

$$
\begin{aligned}
0 & <\left(\int_{0}^{a_{0}} \rho_{0}(x) d x\right)^{\gamma} \\
& =\left(\int_{0}^{a(t)} \rho(x, t) d x\right)^{\gamma} \leq a(t)^{\gamma-1} \int_{0}^{a(t)} \rho^{\gamma} d x,
\end{aligned}
$$

which together with (84), (86), and (91) gives (73). Similarly, we have

$$
\begin{aligned}
0 & <\left(\int_{0}^{y_{0}} \rho_{0}(x) d x\right)^{\gamma} \\
& =\left(\int_{0}^{y(t)} \rho(x, t) d x\right)^{\gamma} \leq y(t)^{\gamma-1} \int_{0}^{y(t)} \rho^{\gamma} d x,
\end{aligned}
$$

which together with (35) implies that

$$
\begin{gathered}
y(t) \geq \begin{cases}c(1+t), & 1<\gamma<2 \alpha, \\
c(1+t)^{1-\gamma}, & \gamma=2 \alpha, \\
c(1+t)^{\alpha /(\gamma-\alpha)}, & \gamma>2 \alpha,\end{cases} \\
-\left[\rho^{\gamma}\right] \leq \begin{cases}c(1+t)^{-1}, & 1<\gamma<2 \alpha, \\
c(1+t)^{-1+\nu}, & \gamma=2 \alpha, \\
c(1+t)^{-\alpha /(\gamma-\alpha)}, & \gamma>2 \alpha .\end{cases}
\end{gathered}
$$

Finally, it follows from (30) that

$$
\rho(a(t), t)=\left((\gamma-\alpha) t+\rho_{0}^{\alpha-\gamma}\left(a_{0}\right)\right)^{-1 /(\gamma-\alpha)},
$$

and as $t$ is large enough, it holds due to (35) that

$$
\begin{aligned}
\rho^{(3 \gamma+2 \alpha-1) / 2}(x, t) & \\
\leq & \rho^{(3 \gamma+2 \alpha-1) / 2}(a(t), t) \\
& +\int_{0}^{a(t)}\left|\left(\rho^{(3 \gamma+2 \alpha-1) / 2}\right)_{x}\right| d x-\left[\rho^{(3 \gamma+2 \alpha-1) / 2}\right] \\
\leq & \rho^{(3 \gamma+2 \alpha-1) / 2}(a(t), t) \\
& +C\left(\int_{0}^{a(t)} \rho^{\gamma} d x\right)^{1 / 2}-C\left[\rho^{\gamma}\right],
\end{aligned}
$$

which together with (86), (91), (95), and (96) gives (75).

\section{Proof of the Main Results}

Proof. The global existence of unique piecewise regular solution to the FBVP (1) and (2) can be established in terms of the short-time existence carried out as in $[1,2,4]$, the uniform apriori estimates, and the analysis of regularities, which indeed follow from Lemmas 5-11. We omit the details. The large time behaviors follow from Lemmas 12-13 directly. The proof of Theorem 2 is completed.

\section{Acknowledgments}

The authors thank the referee for the helpful comments and suggestions on the paper. The research of R. X. Lian is supported by NNSFC no. 11101145, China Postdoctoral Science Foundation no. 2012M520360, Doctoral Foundation of North China University of Water Sources and Electric Power no. 201032, and Innovation Scientists and Technicians Troop Construction Projects of Henan Province. The research of G. J. Zhang is supported by NSF no. GFQQ2460101710.

\section{References}

[1] D. Hoff, "Construction of solutions for compressible, isentropic Navier-Stokes equations in one space dimension with nonsmooth initial data," Proceedings of the Royal Society of Edinburgh A, vol. 103, no. 3-4, pp. 301-315, 1986.

[2] D. Hoff, "Global existence for $1 \mathrm{D}$, compressible, isentropic Navier-Stokes equations with large initial data," Transactions of the American Mathematical Society, vol. 303, no. 1, pp. 169-181, 1987.

[3] D. Hoff, "Global well-posedness of the Cauchy problem for the Navier-Stokes equations of nonisentropic flow with discontinuous initial data," Journal of Differential Equations, vol. 95, no. 1, pp. 33-74, 1992.

[4] D. Hoff, "Spherically symmetric solutions of the Navier-Stokes equations for compressible, isothermal flow with large, discontinuous initial data," Indiana University Mathematics Journal, vol. 41, no. 4, pp. 1225-1302, 1992.

[5] D. Hoff, "Global solutions of the Navier-Stokes equations for multidimensional compressible flow with discontinuous initial data," Journal of Differential Equations, vol. 120, no. 1, pp. 215254, 1995.

[6] D. Hoff, "Strong convergence to global solutions for multidimensional flows of compressible, viscous fluids with polytropic equations of state and discontinuous initial data," Archive for Rational Mechanics and Analysis, vol. 132, no. 1, pp. 1-14, 1995.

[7] G. Q. Chen, D. Hoff, and K. Trivisa, "Global solutions of the compressible Navier-Stokes equations with large discontinuous initial data," Communications in Partial Differential Equations, vol. 25, no. 11-12, pp. 2233-2257, 2000.

[8] D. Hoff, "Discontinuous solutions of the Navier-Stokes equations for multidimensional flows of heat-conducting fluids," Archive for Rational Mechanics and Analysis, vol. 139, no. 4, pp. 303-354, 1997.

[9] D. Hoff, "Dynamics of singularity surfaces for compressible, viscous flows in two space dimensions," Communications on Pure and Applied Mathematics, vol. 55, no. 11, pp. 1365-1407, 2002.

[10] D. Fang and T. Zhang, "Global solutions of the Navier-Stokes equations for compressible flow with density-dependent viscosity and discontinuous initial data," Journal of Differential Equations, vol. 222, no. 1, pp. 63-94, 2006.

[11] R. Lian, Z. Guo, and H. L. Li, "Dynamical behaviors for 1D compressible Navier-Stokes equations with density-dependent viscosity," Journal of Differential Equations, vol. 248, no. 8, pp. 1926-1954, 2010.

[12] R. Lian, J. Liu, H. Li, and L. Xiao, "Cauchy problem for the one-dimensional compressible Navier-Stokes equations," Acta Mathematica Scientia B, vol. 32, no. 1, pp. 315-324, 2012. 
[13] J. F. Gerbeau and B. Perthame, "Derivation of viscous SaintVenant system for laminar shallow water; numerical validation," Discrete and Continuous Dynamical Systems B, vol. 1, no. 1, pp. 89-102, 2001.

[14] F. Marche, "Derivation of a new two-dimensional viscous shallow water model with varying topography, bottom friction and capillary effects," European Journal of Mechanics B, vol. 26, no. 1, pp. 49-63, 2007.

[15] Z. Guo, H. L. Li, and Z. Xin, "Lagrange structure and dynamics for solutions to the spherically symmetric compressible NavierStokes equations," Communications in Mathematical Physics, vol. 309, no. 2, pp. 371-412, 2012.

[16] S. Jiang, Z. Xin, and P. Zhang, "Global weak solutions to $1 \mathrm{D}$ compressible isentropic Navier-Stokes equations with densitydependent viscosity," Methods and Applications of Analysis, vol. 12, no. 3, pp. 239-252, 2005.

[17] T. P. Liu, Z. Xin, and T. Yang, "Vacuum states for compressible flow," Discrete and Continuous Dynamical Systems, vol. 4, no. 1, pp. 1-32, 1998.

[18] M. Okada, Š. Matušů-Nečasová, and T. Makino, "Free boundary problem for the equation of one-dimensional motion of compressible gas with density-dependent viscosity," Annali dell'Università di Ferrara Nuova 8, vol. 48, no. 1, pp. 1-20, 2002.

[19] S.-W. Vong, T. Yang, and C. Zhu, "Compressible Navier-Stokes equations with degenerate viscosity coefficient and vacuum. II," Journal of Differential Equations, vol. 192, no. 2, pp. 475-501, 2003.

[20] T. Yang, Z. A. Yao, and C. Zhu, "Compressible Navier-Stokes equations with density-dependent viscosity and vacuum," Communications in Partial Differential Equations, vol. 26, no. 5-6, pp. 965-981, 2001.

[21] T. Yang and H. Zhao, "A vacuum problem for the onedimensional compressible Navier-Stokes equations with density-dependent viscosity," Journal of Differential Equations, vol. 184, no. 1, pp. 163-184, 2002.

[22] T. Yang and C. Zhu, "Compressible Navier-Stokes equations with degenerate viscosity coefficient and vacuum," Communications in Mathematical Physics, vol. 230, no. 2, pp. 329-363, 2002.

[23] A. Mellet and A. Vasseur, "Existence and uniqueness of global strong solutions for one-dimensional compressible NavierStokes equations," SIAM Journal on Mathematical Analysis, vol. 39, no. 4, pp. 1344-1365, 2008.

[24] Z. Guo, Q. Jiu, and Z. Xin, "Spherically symmetric isentropic compressible flows with density-dependent viscosity coefficients," SIAM Journal on Mathematical Analysis, vol. 39, no. 5, pp. 1402-1427, 2008.

[25] Q. Jiu, Y. Wang, and Z. Xin, "Stability of rarefaction waves to the $1 \mathrm{D}$ compressible Navier-Stokes equations with densitydependent viscosity," Communications in Partial Differential Equations, vol. 36, no. 4, pp. 602-634, 2011.

[26] Q. Jiu and Z. Xin, “The Cauchy problem for 1D compressible flows with density-dependent viscosity coefficients," Kinetic and Related Models, vol. 1, no. 2, pp. 313-330, 2008.

[27] H. L. Li, J. Li, and Z. Xin, "Vanishing of vacuum states and blowup phenomena of the compressible Navier-Stokes equations," Communications in Mathematical Physics, vol. 281, no. 2, pp. 401-444, 2008.

[28] Z. Xin, "Blowup of smooth solutions to the compressible Navier-Stokes equation with compact density," Communications on Pure and Applied Mathematics, vol. 51, no. 3, pp. 229-240, 1998.
[29] D. Hoff and J. Smoller, "Solutions in the large for certain nonlinear parabolic systems," Annales de l'Institut Henri Poincaré. Analyse Non Linéaire, vol. 2, no. 3, pp. 213-235, 1985. 


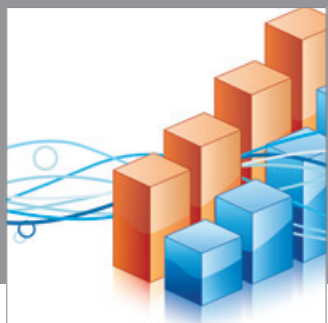

Advances in

Operations Research

mansans

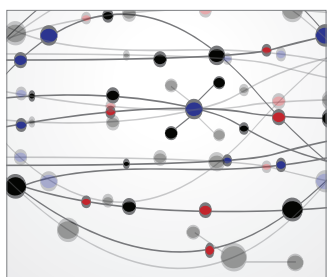

The Scientific World Journal
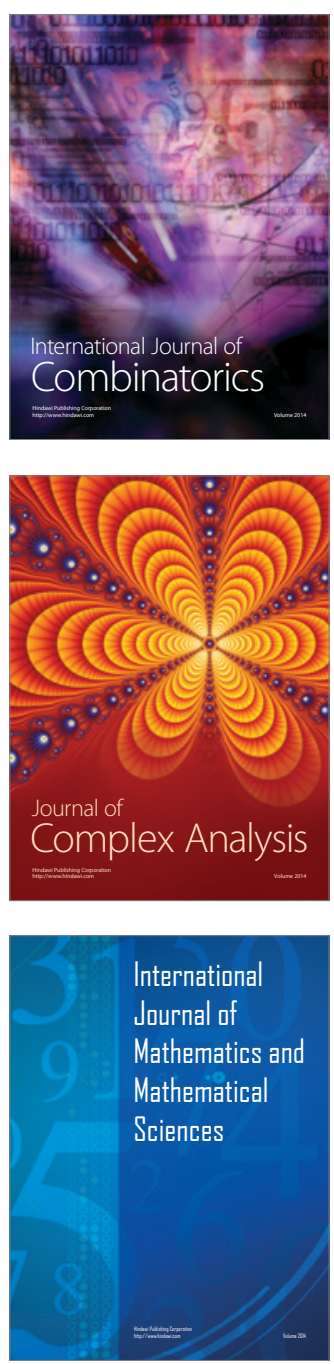
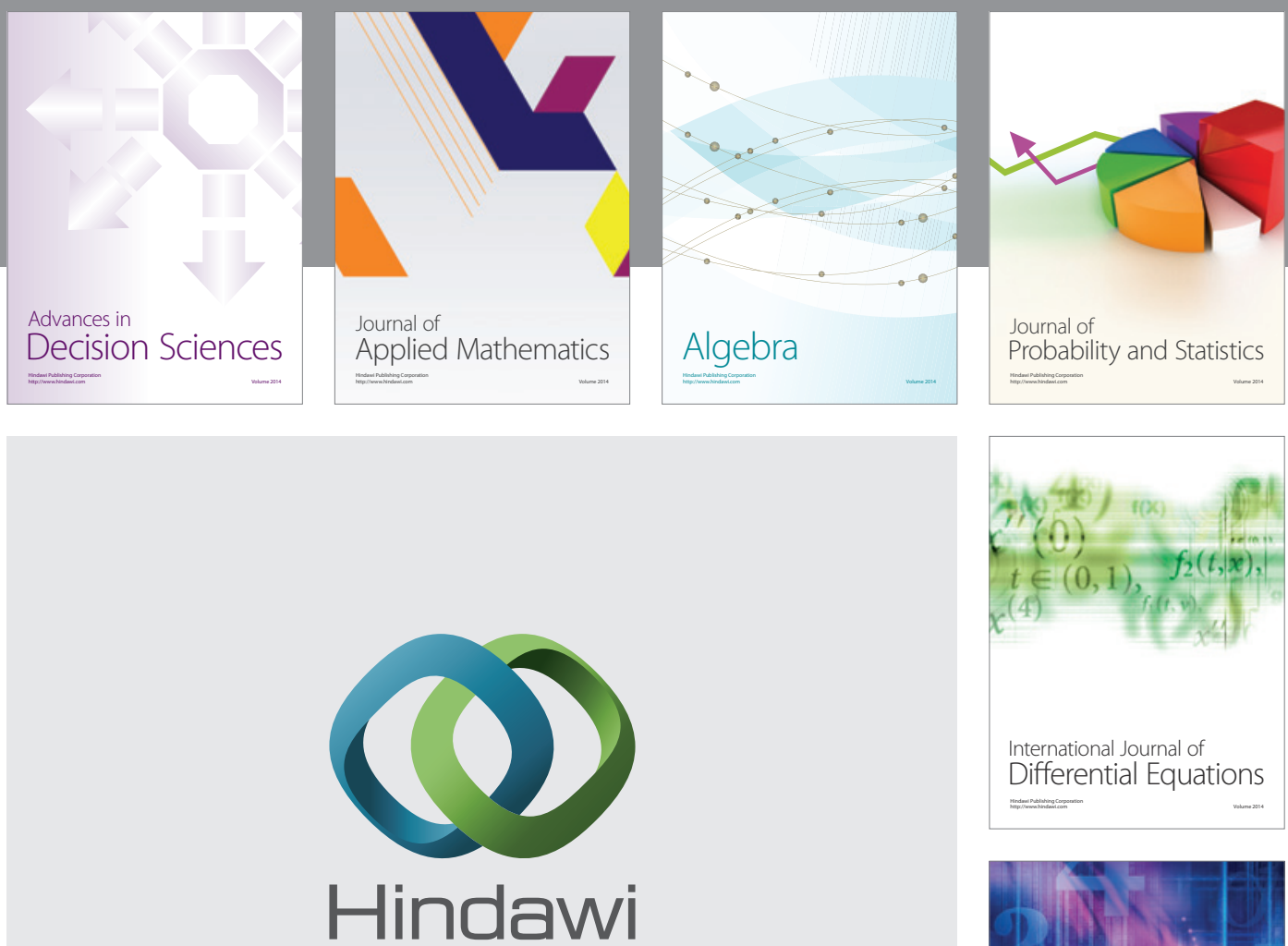

Submit your manuscripts at http://www.hindawi.com
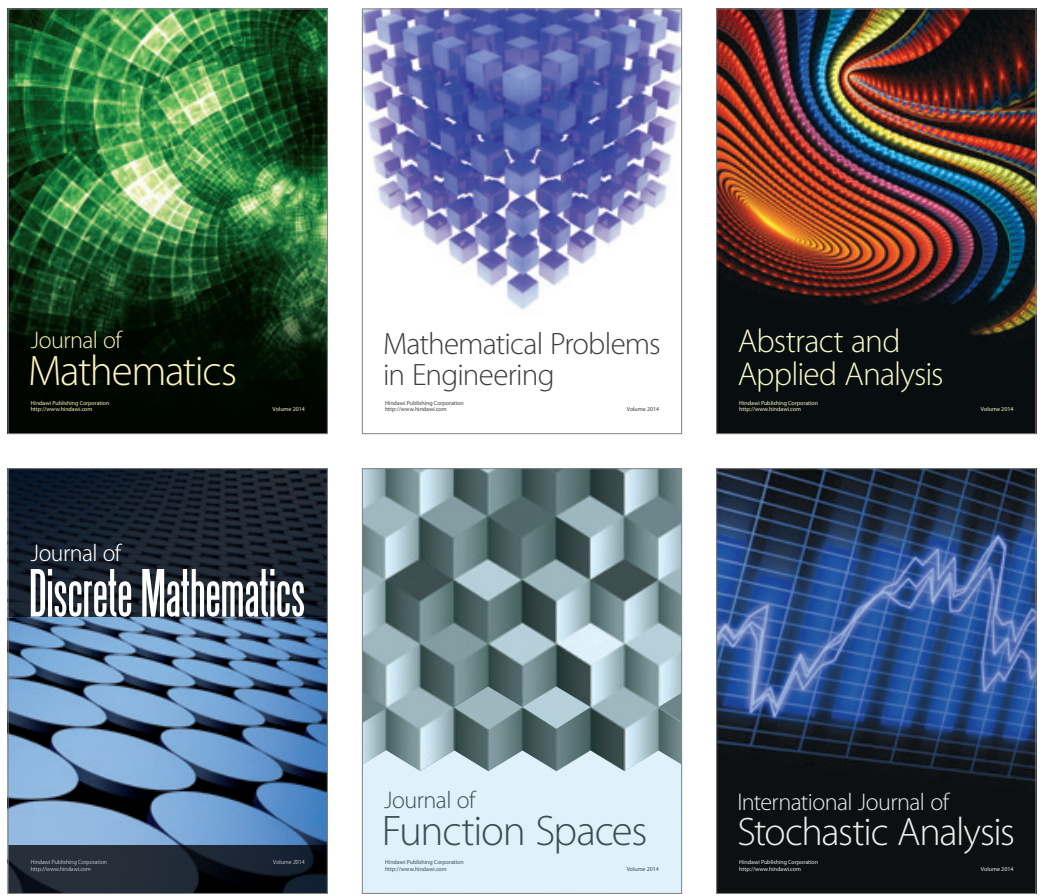

Journal of

Function Spaces

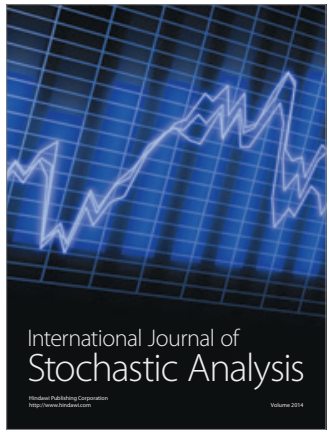

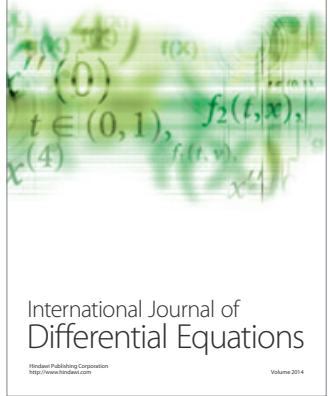
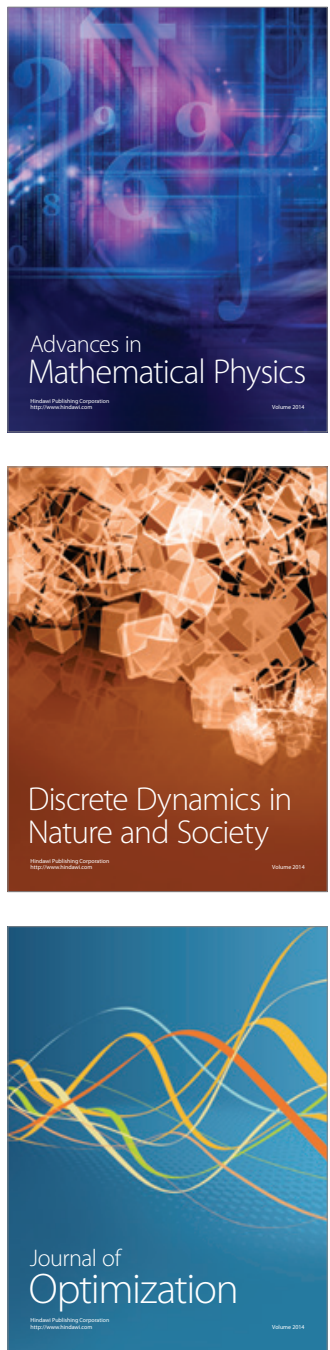